\title{
CLASSIFICATION RELIABILITY FOR GIS-MCDA: AHP AND SENSITIVITY ANALYSIS
}

\author{
Ileana Grave, Víctor Hernández-Díaz, Fidel Serrano-Candela, Tatiana Merino-Benítez, Yosune \\ Miquelajauregui, Luis A. Bojórquez-Tapia
}

\begin{abstract}
One major challenge in Geographical Information Systems-Multicriteria Decision Analysis (GIS-MCDA) is the classification selection to visualize a meaningful spatial pattern. This work proposes a systematic method to (1) select a classification scheme with spatial meaning and to (2) quantify the accuracy of the most sensitive layers, based on a sensitivity analysis. The method is applied to one important aspect of vulnerability assessment (exposure) of the coastal of Yucatán, Mexico where, given a model obtained with the Analytic Hierarchy Process (AHP), the visualizations with meaningful spatial patterns were selected and the most sensitive attributes were identified.
\end{abstract}

Keywords: sensitivity, GIS, data classification methods.

\section{Introduction}

One critical task in map making, to address sustainability issues and concerns, is attaining the appropriate zoning framework of the study region. This task entails classification through Geographical Information Systems-Multicriteria Decision Analysis (GISMCDA). Classification implies a set of abstractions and subjective reasoning involved in the selection of the most meaningful spatial representations (Bojórquez-Tapia et al., 2009). However, classification can be challenging when dealing with highly polysemous concepts that involved multiple viewpoints (Bojórquez-Tapia et al. 2011). Linguistic uncertainty in mapping arises as an outcome of map categories that not only have imprecise meanings but also there meaning is context dependent. Linguistic uncertainty is particularly important in the context of face to face decision-making processes, such as stakeholders' participatory workshops (Carey and Burgman, 2008).

In this paper, we present an approach to evaluate linguistic uncertainty in the context of AHP-based GIS-MCDA. In particular, we address the linguistic uncertainty arising from the land-attributes' performance by means of a sensitivity analysis (Triantaphyllou and Sánchez, 1997). We illustrate this approach through the case of study of coastal vulnerability mapping in Yucatán, México.

\section{Methodology}

1) Vulnerability index. A weighted linear combination is implemented to measure the vulnerability of each basic unit of observation (i.e., polygon or pixel); formally,

$$
V_{i}^{k}=\sum_{j}^{J} w_{i j} x_{i j}^{k}
$$

International Symposium on the Analytic Hierarchy Process 
ISAHP Article: Classification reliability for GIS-MCD: AHP and sensitivity analysis. To Be Submitted to the International Symposium of the Analytic Hierarchy Process 2020, Web Conference.

where $w$ is the weight of attribute $j$, obtained from the AHP, and $x$ is the normalized score of the unit of observation $k$.

2) Classification. The units of observations are classified interactively with stakeholders using different approaches. Because of linguistic uncertainty, there is not a unique classification that conveys the same meaning for all the stakeholders. Therefore, this step entails producing alternative classification schemes that may include different number of categories and different category cuts.

3) Sensitivity analysis. For each classification scheme, the sensitivity test is applied through the threshold value approach (Triantaphyllou \& Sánchez, 1997). Considering the mean value, $\bar{V}_{i}^{c}$, of all the spatial units in each category, $c$, the change in value of a normalized attribute to be included in another category $\bar{V}_{i}^{c_{\rho}}$ is obtained as follows:

$$
\bar{\tau}_{i j}^{c}=\frac{\bar{V}_{i}^{c}-\bar{V}_{i}^{c} \rho}{w_{i j}}, c_{\rho} \in \mathrm{C}=\left\{c_{1}, \ldots, c_{n}\right\}
$$

where $c_{\rho}$ is the reference category from which the probability of switching to another category is estimated.

From eq. (1), the mean normalized score of each land attribute, $\bar{x}_{i j}^{c}$, sets the feasibility range of category switching, $\bar{x}_{i j}^{c}-1 \leq \bar{\tau}_{i j}^{c} \leq \bar{x}_{i j}^{c}$, for attribute $j$, and category $c$. It follows then that the threshold value of categories is

$$
\hat{x}_{i j}^{c}=\overline{\mathrm{x}}_{\mathrm{ij}}^{\mathrm{c}}-\bar{\tau}_{i j}^{c}
$$

Finally, the sensitivity coefficient is calculated by

$$
S_{\mathrm{ij}}^{c}=\frac{1}{\left|\bar{\tau}_{i j}^{c}\right|} \text {. }
$$

4) Fine tuning sensitivity analysis. The category with the highest sensitivity coefficient $c^{*} \in C$ is identified and then is used as input for step (3). Accordingly, for each unit of observation, $k$, contained in $c^{*}$, the value of change, $\tau_{i j}^{k}$, is calculated with (2), where the reference value $V_{i}^{k_{\rho}}$ is taken as the minimum value of the reference class, $c^{\rho}$. Next, the confusion matrix between the categories $c^{*}$ and $c^{\rho}$ is used to estimate the errors of omission and commission.

\section{Case of study: Coastal vulnerability index for Yucatán, México}

The coastal vulnerability index was applied to the coastal zone of Yucatán, México. We implemented de AHP to generate the indices for the three components of vulnerability, namely exposure, sensitivity and resilience. For the sake of clarity, we center on component exposure hereafter. The AHP for exposure entailed the development of a four-level hierarchy to include the biophysical land attributes that determine the vulnerability to hurricanes (Fig. 1). The required pairwise comparisons were elicited in participatory workshops with experts from different disciplines. The AHP produced the importance weights of the attributes, while value functions produced the normalized scores of the units of observations for each attribute.

The exposure index was constructed for each pixel and the initial set of classification methods was taken as equidistant cuts and Weber-Fechner (WF) with different progression factors $(1.3,1.5,1.7,2)$. For 5 categories, the most heterogeneous classification method was WF-1.3, however, a visual inspection reflected the fact that it was necessary to 
ISAHP Article: Classification reliability for GIS-MCD: AHP and sensitivity analysis. To Be Submitted to the International Symposium of the Analytic Hierarchy Process 2020, Web Conference.

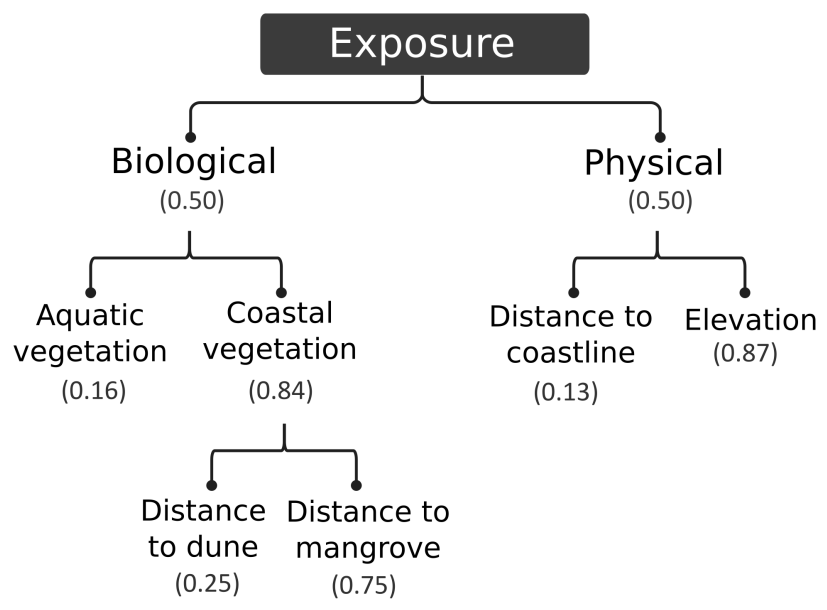

Figure 1. AHP model for exposure to hurricanes

consider fewer classes. The descriptive statistics (Table 1) were obtained for 3 classes, the selected classification methods were the ones with spatial heterogeneity (WF-1.3 and WF1.5), see Figure 2.

The sensitivity test was applied to consider the switching between categories low and high, and medium and high. The classification with the lowest sensitivity was WF-1.5 (Table 2). In terms of attributes, most sensitives are distance to mangrove and elevation.

Fine tuning was applied to the attribute distance to mangrove for WF-1.5. The number of pixels with feasible rank reversal (Figure 2) and the confusion matrix (Table 3) were obtained. Classification errors were calculated for the cases of feasible category switching with $s_{\mathrm{ij}}^{C}>5$. The categorization presented an error of commission of $17 \%$ and an error of omission of $16 \%$ (Table 3 ).

Table 1. Descriptive statistics for each progression factor for 3 classes

\begin{tabular}{rlrrrrr}
\hline \hline $\begin{array}{l}\text { Progression } \\
\text { factor }\end{array}$ & Class & $\begin{array}{c}\text { Number of } \\
\text { pixels }\end{array}$ & \multicolumn{1}{c}{ Min } & max & mean & \multicolumn{1}{c}{ median } \\
\hline 1.3 & Low & 44668 & 0.307 & 0.717 & 0.658 & 0.675 \\
& Medium & 14126 & 0.717 & 0.84 & 0.777 & 0.776 \\
& High & 38870 & 0.84 & 1 & 0.950 & 0.968 \\
\hline 1.5 & Low & 7000 & 0.307 & 0.615 & 0.589 & 0.598 \\
& Medium & 43917 & 0.615 & 0.769 & 0.681 & 0.68 \\
& High & 46747 & 0.769 & 1 & 0.926 & 0.948 \\
\hline 1.7 & Low & 521 & 0.307 & 0.546 & 0.522 & 0.53 \\
& Medium & 43793 & 0.547 & 0.714 & 0.659 & 0.675 \\
& High & 53350 & 0.714 & 1 & 0.903 & 0.928 \\
\hline 2 & Low & 26 & 0.307 & 0.479 & 0.431 & 0.455 \\
& Medium & 14454 & 0.481 & 0.653 & 0.613 & 0.616 \\
& High & 83184 & 0.653 & 1 & 0.823 & 0.817 \\
\hline \hline
\end{tabular}

International Symposium on the Analytic Hierarchy Process
DEC. 3 - DEC. 6, 2020 
ISAHP Article: Classification reliability for GIS-MCD: AHP and sensitivity analysis. To Be Submitted to the International Symposium of the Analytic Hierarchy Process 2020, Web Conference.

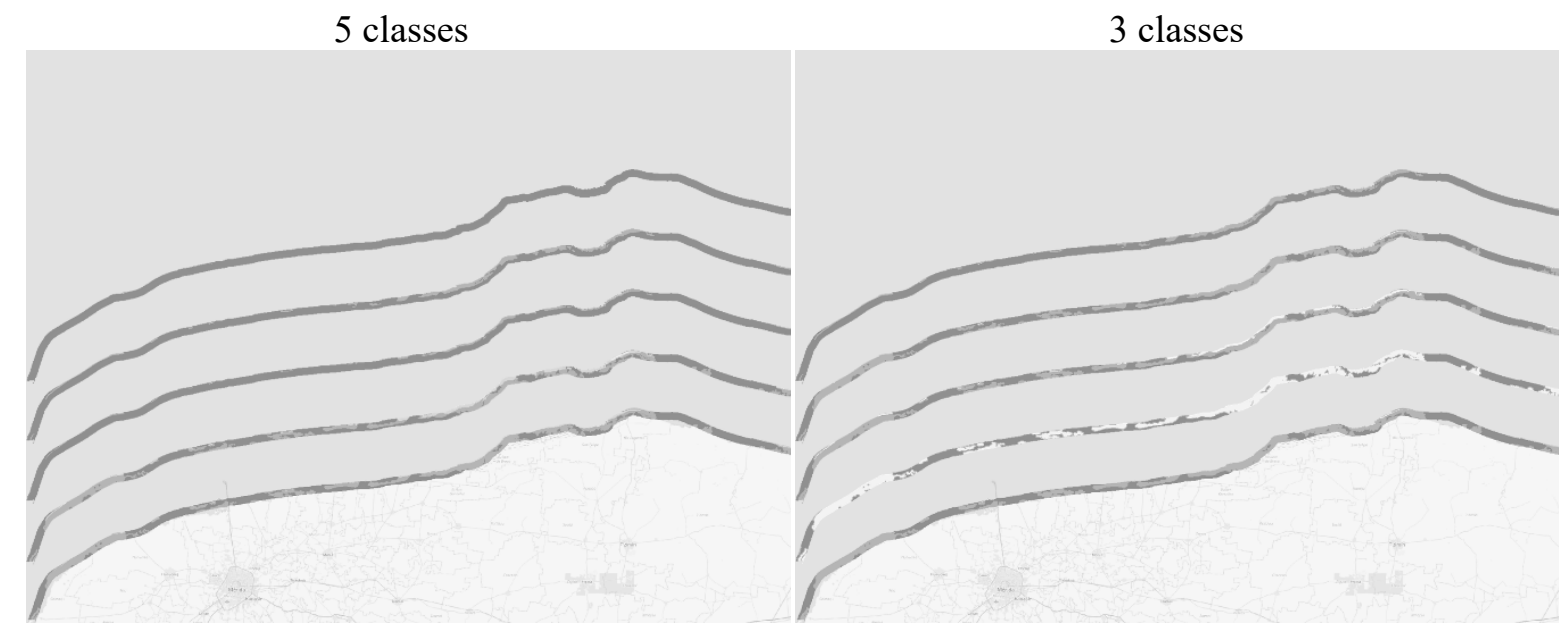

Figure 2. Exposure maps for different classification methods. From bottom to up: equidistant, Weber-Fechner (WF): 1.3, 1.5, 1.7, 2.0

Table 2. Sensitivity coefficient

\begin{tabular}{|c|c|c|c|c|c|c|c|}
\hline \multirow{2}{*}{$\begin{array}{l}\text { Progression } \\
\text { factor }\end{array}$} & \multicolumn{2}{|c|}{$\begin{array}{l}\text { Category } \\
\text { switching }\end{array}$} & \multicolumn{5}{|c|}{ Attribute } \\
\hline & From & to & $\begin{array}{c}\text { Aquatic } \\
\text { vegetation }\end{array}$ & $\begin{array}{l}\text { Distance to } \\
\text { mangrove }\end{array}$ & $\begin{array}{l}\text { Distance } \\
\text { to dune }\end{array}$ & Elevation & $\begin{array}{c}\text { Distance to } \\
\text { coastline }\end{array}$ \\
\hline \multirow[t]{4}{*}{1.3} & Low & High & $\mathrm{NF}$ & 1.079 & NF & $\mathrm{NF}$ & $\mathrm{NF}$ \\
\hline & Medium & High & $\mathrm{NF}$ & 1.821 & NF & NF & NF \\
\hline & High & Low & NF & NF & NF & 1.490 & NF \\
\hline & High & Medium & $\mathrm{NF}$ & 1.821 & NF & 2.513 & NF \\
\hline \multirow[t]{4}{*}{1.5} & Low & High & $\mathrm{NF}$ & NF & NF & NF & NF \\
\hline & Medium & High & $\mathrm{NF}$ & 1.285 & NF & $\mathrm{NF}$ & NF \\
\hline & High & Low & $\mathrm{NF}$ & NF & NF & 1.290 & NF \\
\hline & High & Medium & NF & 1.285 & NF & 1.776 & NF \\
\hline
\end{tabular}

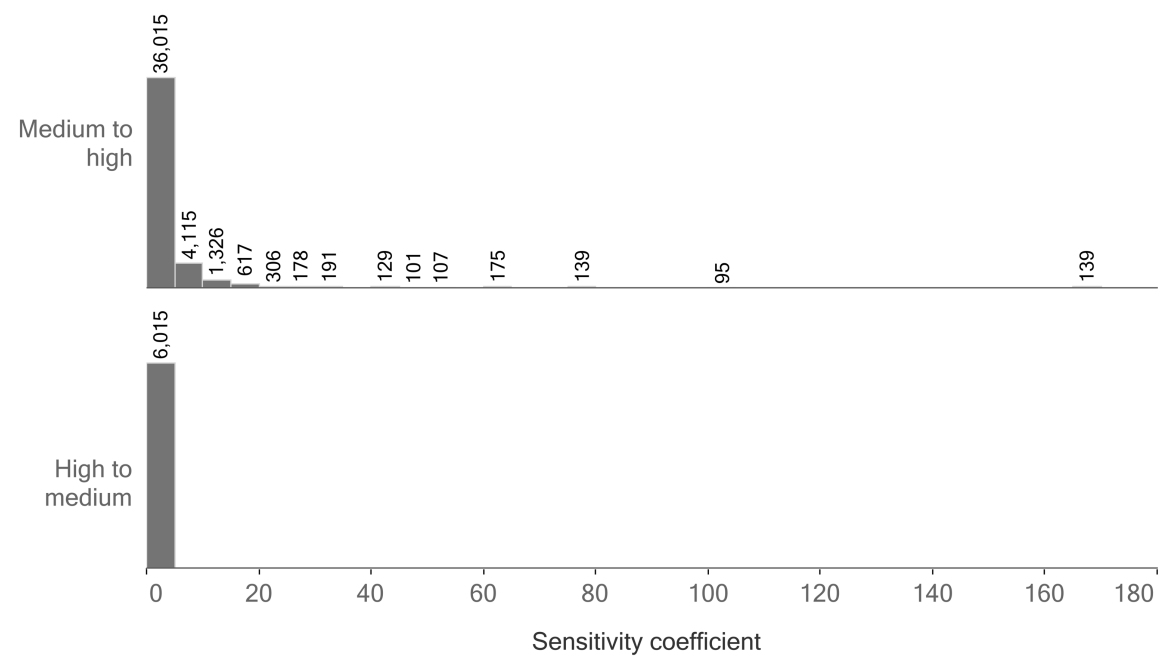

Figure 3. Distance to mangrove feasible category switching

International Symposium on the Analytic Hierarchy Process 
ISAHP Article: Classification reliability for GIS-MCD: AHP and sensitivity analysis. To Be Submitted to the International Symposium of the Analytic Hierarchy Process 2020, Web Conference.

Table 3. Confusion matrix elements for distance to mangrove

Reference class

\begin{tabular}{|c|c|c|c|c|}
\hline \multirow{3}{*}{$\begin{array}{r}\text { Switching } \\
\text { class }\end{array}$} & & Medium & High & \multirow{3}{*}{$\begin{array}{l}17 \% \\
0 \%\end{array}$} \\
\hline & \multirow{3}{*}{$\begin{array}{c}\text { Medium } \\
\text { High }\end{array}$} & 36299 & 7618 & \\
\hline & & 0 & 46747 & \\
\hline & & $0 \%$ & $16 \%$ & \\
\hline
\end{tabular}

\section{Discussion and Conclusions}

The linguistic uncertainty inherent to the participatory workshop was addressed by applying sensitivity analysis to the land classifications. Results proved to be useful to the stakeholders in identifying the classification scheme that best conveyed the coastal zone's differential exposure to hurricanes.

During the workshops, the participants first deliberated whether the 3-category map gave a better representation than the 5-category one. The participants selected the former and then deliberated on which of two classifications (WF-1.3 and WF-1.5) was the best. The sensitivity analysis provided the justification for selecting classification WF-1.5. The reason underlying this selection was that classification WF-1.5 had the lowest sensitivity coefficients and the least category switching (4 changes). Fine tuning through the error of commission revealed that classification WF-1.5 to hurricanes may be underestimated in $17 \%$ of the coastal zone area.

Even though the approach presented here was applied to vulnerability indicators, it can be implemented to any AHP-based GIS-MCDA to (1) identify how measurement errors of land attributes affect the classification of maps, and (2) select the nominal map that conveys the best representation of a geographic phenomenon.

Acknowledgments. This work was supported by UNAM PAPIIT-IV100118; ASUUNAM Binational Laboratory of Sustainability, Vulnerability, and Adaptation to Climate Change; FOMIX YUC-2018-04-01-88958. TMB acknowledges UNAM's Posgrado en Ciencias de la Sostenibilidad and the support of CONACYT-1003060.

\section{Key References}

Bojórquez-Tapia, L. A., Cruz-Bello, G. M., \& Luna-González, L. (2013). Connotative land degradation mapping: A knowledge-based approach to land degradation assessment. Environmental Modelling and Software, 40, 51-64.

Bojórquez-Tapia, L.A., Cruz-Bello, G.M., Luna-González, L., Juárez, L., Ortiz-Pérez, M.A., 2009. V-DRASTIC: Using visualization to engage policymakers in groundwater vulnerability assessment. J. Hydrol. 373, 242-255.

Carey, J. M., \& Burgman, M. A. (2008). Linguistic uncertainty in qualitative risk analysis and how to minimize it. Annals of the New York Academy of Sciences, 1128(1), 1317.

Triantaphyllou, E., \& Sánchez, A. (1997). A Sensitivity Analysis Approach for Some Deterministic Multi-Criteria Decision-Making Methods. Decision Sciences, 28(1), 151-194. 